\title{
SIMULAÇÃO COMO ESTRATÉGIA DE ENSINO-APRENDIZAGEM NO TRATAMENTO DE FERIDAS: RELATO DE EXPERIÊNCIA
}

\section{SIMULATION AS A TEACHING-LEARNING STRATEGY IN WOUND TREATMENT: EXPERIENCE REPORT}

\section{SIMULACIÓN COMO ESTRATEGIA DE ENSEÑANZA-APRENDIZAJE EN EL TRATAMIENTO DE HERIDAS: INFORME DE EXPERIENCIA}

\author{
Alice Molonha ${ }^{1}$ \\ Bruna de Oliveira Fakhr ${ }^{2}$ \\ Felipe Amato Custódio ${ }^{3}$ \\ Thiago Pereira Lopes ${ }^{4}$ \\ Simone Roecker \\ Juliane Pagliari Araujo
}

\begin{abstract}
Como citar este artigo: Molonha A, Fakhr BO, Custódio FA, Lopes TP, Roecker S, Araujo JP. Simulação como estratégia de ensino-aprendizagem no tratamento de feridas: relato de experiência. Rev baiana enferm. 2020;34:e37039.

Objetivo: relatar a experiência de estudantes do curso técnico em enfermagem sobre a utilização da simulação como estratégia de ensino-aprendizagem no tratamento de feridas. Método: relato de experiência de simulação no tratamento de feridas como estratégia de ensino-aprendizagem. Participaram 25 estudantes e 2 docentes de um curso técnico em enfermagem em um município paranaense. A simulação foi realizada nos meses de novembro e dezembro de 2019. Resultados: a simulação aplicada no tratamento de feridas desenvolveu-se em sete etapas. No decorrer das etapas, os participantes estudaram sobre feridas e prática de realização de curativos, confeccionaram as feridas e realizaram a simulação do cuidado. Conclusão: a experiência revelou que este método oferece benefícios no processo ensino-aprendizagem, favorece aos estudantes a oportunidade de uma atitude proativa na construção do conhecimento, bem como habilita-os e aproxima-os da prática profissional.
\end{abstract}

Descritores: Educação em Enfermagem. Ferimentos e Lesões. Ensino. Simulação.

Objective: to report the experience of nursing technical students on the use of simulation as a teaching-learning strategy in wound treatment. Method: report of simulation experience in wound treatment as a teaching-learning strategy. The participants were 25 students and 2 professors of a nursing technical course in a city of Paraná. The simulation was carried out in November and December 2019. Results: the simulation applied in the treatment of wounds was developed in seven steps. During the steps, the participants studied wounds and practice of dressings, made the wounds and performed the care simulation. Conclusion: the experience revealed that this method offers

\footnotetext{
Discente do Curso Técnico de Enfermagem. Instituto Federal do Paraná. Londrina, Paraná, Brasil. https://orcid.org/0000-0002-1098-1952. Discente do Curso Técnico de Enfermagem. Instituto Federal do Paraná. Londrina, Paraná, Brasil. https://orcid.org/0000-0002-4288-0830. Discente do Curso Técnico de Enfermagem. Instituto Federal do Paraná. Londrina, Paraná, Brasil. https://orcid.org/0000-0003-0635-I242.

Discente do Curso Técnico de Enfermagem. Instituto Federal do Paraná. Londrina, Paraná, Brasil. https://orcid.org/0000-0002-5398-3886. Enfermeira. Doutora em Saúde Coletiva. Docente do Instituto Federal do Paraná. Londrina, Paraná, Brasil. https://orcid.org/0000-000 I-98 I 2-720X.

Enfermeira. Mestre em Biociências e Saúde. Docente do Instituto Federal do Paraná. Londrina, Paraná, Brasil. juliane.pagliari@ifpr.edu.br. https://orcid.org/0000$0001-7821-6731$.
} 
benefits in the teaching-learning process, favors students the opportunity of a proactive attitude in the construction of knowledge, as well as enables and approaches them to professional practice.

Descriptors: Education, Nursing. Wounds and Injuries. Teaching. Simulation Technique.

Objetivo: informar la experiencia de los estudiantes técnicos de enfermería sobre el uso de la simulación como estrategia de enseñanza-aprendizaje en el tratamiento de heridas. Método: informe de experiencia de simulación en el tratamiento de heridas como estrategia de enseñanza-aprendizaje. Los participantes fueron 25 estudiantes y 2 profesores de un curso técnico de enfermería en un municipio de Paraná. La simulación se llevó a cabo en noviembre y diciembre de 2019. Resultados: la simulación aplicada en el tratamiento de heridas se desarrolló en siete etapas. Durante las etapas, los participantes estudiaron heridas y practicaron curativos, realizaron las heridas y la simulación del cuidado. Conclusión: la experiencia revela que este método ofrece beneficios en el proceso de enseñanza-aprendizaje, favorece a los estudiantes la oportunidad de una actitud proactiva en la construcción del conocimiento, así como les permite y los acerca a la práctica profesional.

Descriptores: Educación en Enfermería. Heridas y Traumatismos. Enseñanza. Simulación.

\section{Introdução}

O processo formativo em saúde e em enfermagem deve ser discutido e rediscutido permanentemente, considerando as características do mundo atual do trabalho, assim como as necessidades da adequação do setor de saúde em relação às novas realidades existentes ${ }^{(1)}$. Destaca-se que esse processo é dinâmico e está em constante transformação. Desse modo, o ato de ensinar e aprender, na era das comunicações ultrarrápidas e das fake news, precisa ser repensado e demanda métodos inovadores, que visem culminar numa prática pedagógica ética, crítica, reflexiva e transformadora ${ }^{(2)}$.

No que tange ao processo ensino-aprendizagem, salienta-se que o estudante deve ser tido como o ator principal, para que a aprendizagem permeie a sua vida e ocupe um papel importante. Muitos educadores definem a aprendizagem como elemento de mudança comportamental, a qual deriva de experiências vividas pelo sujeito. O estilo de aprendizagem é singular e varia de acordo com a percepção, estrutura cognitiva, emocional e fisiológica de cada indivíduo ${ }^{(3)}$.

O ensino de enfermagem, assim como ocorre em outros cursos da área da saúde, tem buscado frequentemente um aprendizado efetivo, que ofereça bagagem técnica-científica ao longo de toda formação ${ }^{(4)}$. O uso de metodologias ativas, como a simulação, tem integrado cada vez mais a formação da enfermagem.
Nesse sentido, a simulação é apresentada como um processo dinâmico, o qual envolve a criação de uma situação hipotética e que incorpora uma representação autêntica da realidade. Permite também a participação ativa do estudante, integrando as complexidades da aprendizagem prática e teórica com a oportunidade de repetição, feedback, avaliação e reflexão, reduzindo eventos adversos. Podem contribuir para o aumento da autoconfiança, bem como para a redução da ansiedade e do medo em diferentes faixas etárias e diferentes níveis educacionais ${ }^{(3-4)}$.

Estudo realizado em Salvador (BA) mostrou que a simulação com o uso de manequins permite que estudantes aprimorem suas habilidades, pois é um instrumento de ensino e avaliação que traz vantagens para o aprendizado de práticas básicas e complexas. Na enfermagem, entre os diversos procedimentos que requerem o treinamento, encontra-se a técnica de curativo no tratamento de feridas, tornando assim fundamental o ensino dos estudantes de forma simulada ${ }^{(5)}$.

Diante desse cenário, tem-se discutido sobre o papel da simulação na formação do enfermeiro. No entanto, pouco ou nada se tem com relação à formação do técnico em enfermagem. Estudantes de diferentes níveis educacionais da área da saúde podem ser beneficiados pelo método de ensino por meio da simulação, como 
oportunidade de aprendizagem holística reflexiva, que integra a teoria com a prática.

Nesse contexto, o presente estudo teve por objetivo relatar a experiência de estudantes do curso técnico em enfermagem sobre a utilização da simulação como estratégia de ensino-aprendizagem no tratamento de feridas.

\section{Método}

Trata-se de um relato descritivo de experiência de simulação no tratamento de feridas como estratégia de ensino-aprendizagem junto aos estudantes do curso técnico em enfermagem. Salienta-se que este tipo de estudo permite descrever as situações vivenciadas pelos atores, reforçando a importância do feito na construção e remodelação dos saberes científicos e populares ${ }^{(6)}$. A atividade contou com 25 estudantes e 2 docentes de um curso técnico em enfermagem em um município do Norte do estado do Paraná.

Essa ação ocorreu integrada a um componente curricular denominado "Fundamentos de Enfermagem II" e a um projeto de extensão intitulado "365 dias promovendo saúde e cuidados" cadastrado no Comitê de Pesquisa e Extensão institucional.

A ação foi realizada nos meses de novembro e dezembro de 2019, quase ao final do componente curricular. Nesse momento, os estudantes já haviam tido uma aproximação com feridas e curativos. O cenário da ação foi o laboratório de enfermagem do Instituto Federal do Paraná, campus Londrina. Salienta-se que esse laboratório foi organizado para atender especificamente essa atividade, não sendo um laboratório de práticas de simulação realística. Os docentes alocaram materiais e equipamentos para melhor reproduzir a criação do cenário de uma enfermaria e/ou sala de atendimento.

A construção dessa simulação surgiu da inquietação das docentes sobre as dificuldades de entendimento dos estudantes e sobre a falta de execução técnica na realização de curativos de feridas crônicas e de grande extensão. As docentes reuniram-se e refletiram sobre um método de abordar essa temática, em que fosse efetiva a compreensão dos estudantes.

Assim, a ação de simulação permeou sete etapas que estão detalhadamente descritas na seção resultados, que seguiram desde as orientações (Briefing ou Prebriefing) até a discussão e reflexão acerca da temática e avaliação da atividade (Debriefing), seguida pela apresentação e divulgação a toda a comunidade acadêmica.

\section{Resultados}

A simulação aplicada no tratamento de feridas transcorreu em sete etapas. No primeiro encontro, o tema sobre feridas crônicas e/ou de grande extensão foi abordado com os estudantes. Nesse momento, discutiu-se e refletiu-se sobre tipos de lesão, tecidos, exsudatos, entre outros aspectos. No segundo momento, os participantes aprenderam sobre técnicas de curativo em atividades no laboratório de enfermagem. No terceiro encontro, discutiu-se sobre simulação no cuidado de feridas. Os estudantes foram divididos em cinco grupos de quatro ou cinco participantes cada um, os quais ficaram responsáveis pela construção de feridas, para, posteriormente, discutir e confeccionar curativos com o grande grupo.

Nas três etapas iniciais, no caso, denominadas como Briefing ou Prebriefing, antecedendo a simulação, são disponibilizadas orientações e informações aos participantes de forma a prepará-los para o desenvolvimento da experiência da simulação. Pretende-se esclarecer os objetivos do cenário, bem como transmitir orientações para o uso dos equipamentos, dos manequins, além do tempo para a simulação ${ }^{(7)}$.

Após o término das primeiras etapas, os participantes reuniram-se para a construção das feridas, o que configurou a quarta etapa. As feridas foram construídas com materiais escolhidos pelos grupos (algodão, tinta, maquiagem, corantes e farinhas culinárias, cola, entre outros) e os locais das lesões também foram de livre escolha, conforme Figura 1: 
Figura 1 - Feridas construídas pelos estudantes para a prática de simulação

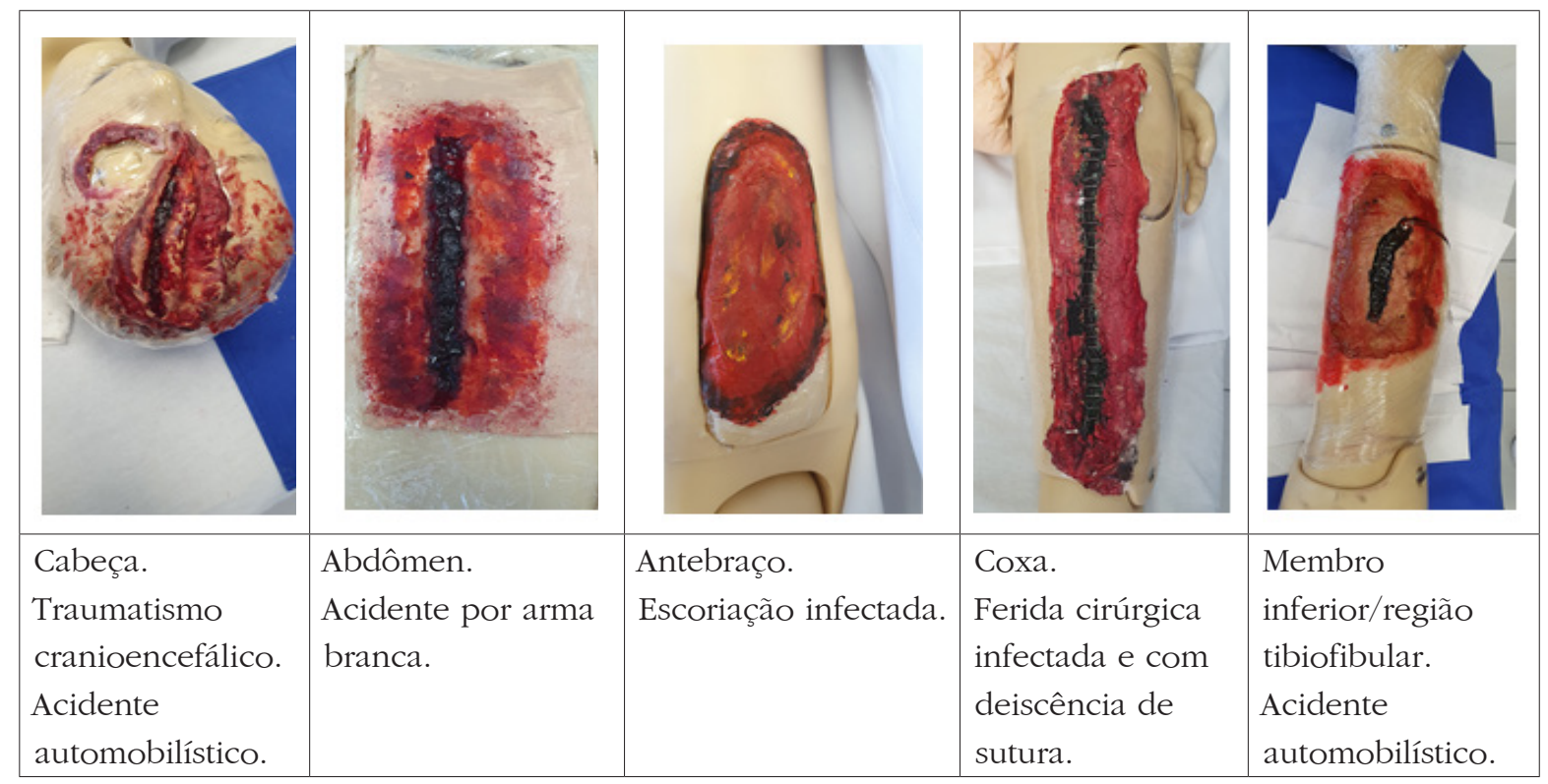

Fonte: Elaboração própria.

$\mathrm{Na}$ etapa cinco, em data previamente agendada, ocorreu a atividade de simulação de curativos. Cada grupo ficou responsável por organizar essa atividade e aplicar a simulação para os colegas. Os grupos apresentaram o histórico da ferida, como foi causada, há quanto tempo, os tecidos que estavam expostos e o tratamento que seria realizado.

Além da aplicação de curativos, realizados com soro fisiológico, gaze e aplicação de medicações prescritas, os docentes apresentaram diversos curativos e coberturas especiais, que podem ser aplicados em situações específicas. Outro tratamento apresentado pelas docentes foi o curativo a vácuo ou terapia por pressão negativa, o qual apresenta-se como um importante método no tratamento das feridas complexas e tem como proposta principal acelerar o processo de reparação e preparo do leito da ferida até sua cobertura definitiva ${ }^{(8)}$.

Ressalta-se que o técnico em enfermagem somente executa o tratamento prescrito por profissionais médicos e/ou enfermeiros. Assim, como o técnico em enfermagem não realiza a prescrição de tratamento de feridas, os estudantes apresentaram possíveis tratamentos, os quais foram discutidos no grande grupo. Cada simulação durou, em média, 30 minutos.
A sexta etapa consistiu em reunir os estudantes para discussão e reflexão acerca da temática e avaliação da atividade. Foi utilizada a técnica do Debriefing, que é um momento de reflexão pós-experiência, realizado imediatamente após a simulação, com o objetivo de refletir sobre a prática, a fim de consolidar e efetivar a aprendizagem dos discentes ${ }^{(9)}$.

$\mathrm{Na}$ sétima e última etapa, os discentes expuseram, para a comunidade acadêmica, as feridas construídas, que ficaram expostas durante quatro dias. Foram enviados convites para a visitação, a fim de dar visibilidade à ação e também para que pessoas de outras áreas pudessem conhecer o trabalho da enfermagem e as diferentes metodologias de ensino-aprendizagem adotadas pelo curso técnico em enfermagem.

A realização de simulação no processo ensino-aprendizagem vem ganhando força nos últimos anos, em decorrência dos resultados positivos apresentados pelos docentes e também pelos estudantes de diversas áreas da saúde ${ }^{(3-4)}$.

Ao longo dessa atividade, os participantes puderam expor suas dúvidas, discutir e treinar sobre os melhores métodos de curativo em cada caso. Visualizar os diferentes tecidos e exsudatos foi de grande valia, pois o uso somente de figuras dispostas em apresentação de slides 
ou livros, muitas vezes, não é suficiente para a fixação do conteúdo.

Durante o processo de simulação, para alcançar melhor resultado com a utilização, disseminação e aplicação do método, faz-se necessário estabelecer correlação com a prática diária e acrescentar outros elementos que se julguem importantes ${ }^{(10)}$. As docentes foram mediadoras das simulações, a fim de proporcionar um resultado satisfatório na atividade proposta. Dessa maneira, a ação gerou impacto e formação do cuidado em feridas crônicas e/ou de grande extensão realizado pelo técnico em enfermagem.

Essa experiência de realizar simulações de cuidados de feridas de grande extensão e/ou crônicas possibilitou aos estudantes do curso técnico em enfermagem a percepção da necessidade de ampliar essas atividades para outros cuidados. A segurança do paciente, entendida como a redução do risco de dano associado à atenção à saúde ${ }^{(11)}$ a um mínimo aceitável, vem sendo muito discutida no desenvolvimento do cuidado integral. Assim, esse desafio global deve ser inserido nas atividades e discussões locais. Tem-se o entendimento de que o uso de simulações pode colaborar para implementar o "pensamento" de segurança do paciente no tratamento de feridas, bem como para a demanda por treinamentos e atualizações dos profissionais técnicos em enfermagem, em sua atuação no mundo do trabalho.

Em relação às dificuldades experienciadas, ressalta-se a falta de um cenário próprio de simulação, para que a atividade proporcionasse maior realidade, além da aproximação à prática vivenciada. No entanto, observou-se que essa dificuldade foi superada, por meio de adequações dos espaços e cenários disponíveis, tornando possível a realização da simulação de forma satisfatória.

Estudo apontou também a dificuldade da elaboração de um cenário que possibilitasse a vivência da realidade, visto que muitos docentes não dispunham de tempo para tal elaboração, bem como muitas instituições de ensino não disponibilizavam locais e materiais para essas atividades ${ }^{(12)}$.

Outro ponto que necessita ser explorado é que a simulação ainda não faz parte da rotina educacional de nível técnico. Assim, alguns discentes não ficaram à vontade para simular, não interagindo de forma efetiva com os demais colegas. Autores afirmam que é recomendado fornecer subsídios, para que os estudantes incorporem o personagem, sendo indicado um roteiro preestabelecido para atender aos objetivos definidos $^{(10)}$.

Salienta-se que a simulação não substitui atividades de campo na formação do estudante do curso técnico em enfermagem, mas pode agregar e substituir métodos tradicionais de ensino, que não condizem com o novo perfil de estudantes. Por isso, o docente deve ter como dever sensibilizar o interesse do estudante em aprender e compreender o processo do cuidado na enfermagem.

\section{Conclusão}

A experiência revelou que a estratégia de simulação no tratamento de feridas oferece vários benefícios no processo ensino-aprendizagem, dentre os quais destaca-se o fato de favorecer ao estudante a oportunidade de vivenciar uma atitude proativa na construção do conhecimento, com o envolvimento entre docentes e estudantes. Dessa forma, torna-se imprescindível que docentes busquem metodologias que proporcionem um ensino mais atrativo, sempre primando por um ensino inovador e significativo.

Conclui-se que a simulação do procedimento de feridas associada ao curativo demonstrou-se vantajosa para o processo de ensino-aprendizagem do curso técnico em enfermagem, como uma estratégia capaz de habilitar e aproximar os estudantes da prática profissional, a fim de que possam prestar uma assistência de qualidade aos pacientes em sua atuação profissional.

Assim, a divulgação dessa atividade de simulação do curso técnico em enfermagem tem como intuito fomentar que outros docentes e instituições de ensino técnico percebam a possibilidade de realizar essa estratégia de ensino e possam adotar a simulação como realidade. 


\section{Colaborações:}

1 - concepção, projeto, análise e interpretação dos dados: Simone Roecker e Juliane Pagliari Araujo;

2 - redação do artigo e revisão crítica relevante do conteúdo intelectual: Alice Molonha, Bruna de Oliveira Fakhr, Felipe Amato Custódio, Thiago Pereira Lopes, Simone Roecker e Juliane Pagliari Araujo;

3 - aprovação final da versão a ser publicada: Simone Roecker e Juliane Pagliari Araujo.

\section{Referências}

1. Costa RRO, Medeiros SM, Martins JCA, Cossi MS, Araújo MS. Percepção de estudantes da graduação em enfermagem sobre a simulação realística. Rev cuid. 2017;8(3):1799-808. DOI: http://dx.doi. org/10.15649/cuidarte.v8i3.425

2. Veiga GA, Araújo MC, Cauduro FLF, Andrade J. Metodologia ativa no estágio supervisionado de enfermagem: inovação na atenção primária à saúde. Rev baiana enferm. 2020;34:e34857. DOI: http://dx.doi.org/10.18471/rbe.v34.34857

3. Nascimento MS, Magro MCS. Simulação realística: método de melhoria de conhecimento e autoconfiança de estudantes de enfermagem na administração de medicamento. REME - Rev Min Enferm. 2018;22:e-1094. DOI: 10.5935/ $1415-2762.20180024$

4. Marcomini EK, Martins ESM, Lopes NV, Paula NVK, Liberati BAS. Influência da simulação realística no ensino e aprendizado da enfermagem. Rev Varia Scientia - Ciênc Saúde [Internet]. 2017 [cited2020Mar23];3(2):233-40.Availablefrom:http:// e-revista.unioeste.br/index.php/variasaude/ article/view/17687/12260
5. Jesus BC, Ramos GF, Silva CCR, Gomes VCO, Silva GTR. Simulação em manequins como estratégia de ensino-aprendizagem para avaliação de ferida: relato de experiência. ESTIMA. 2017;15(4):245-9.DOI:10.5327/Z1806-3144201700040009

6. Minayo MCS. O desafio do conhecimento: pesquisa qualitativa em saúde. São Paulo: Hucitec; 2013.

7. Meakim C, Boese $\mathrm{T}$, Decker S, Franklin AE, Gloe D, Lioce L, et al. Standards of Best Practice: Simulation Standard I: Terminology. Clin Simulation Nurs. 2013;9(Supl 6):S3-S11. DOI: http://dx.doi.org/10.1016/j.ecns.2013.04.001

8. Lima RVKS, Coltro PS, Farina Júnior JA. Terapia por pressão negativa no tratamento de feridas complexas. Rev Col Bras Cir. 2017 fev;44(1):81-93. DOI: https://doi.org/10.1590/0100-69912017001001

9. Reed SJ. Written debriefing: evaluating the impact of the addition of a written component when debriefing simulations. Nurse Educ Pract. 2015;15(6):543-8. DOI: 10.1016/j.nepr.2015.07.011

10. Kaneko RMU, Lopes MHBM. Realistic health care simulation scenario: what is relevant for its design? Rev esc enferm USP. 2019;53:e03453. DOI: http:// dx.doi.org/10.1590/S1980-220X2018015703453

11. Brasil. Agência Nacional de Vigilância Sanitária. Pacientes pela segurança do paciente em serviços de saúde: Como posso contribuir para aumentar a segurança do paciente? Orientações aos pacientes, familiares e acompanhantes. Brasília, DF; 2017.

12. Garbuio DC, Oliveira ARS, Kameo SY, Melo ES, Dalri MCB, Carvalho EC. Simulação clínica em enfermagem: relato de experiência sobre a construção de um cenário. Rev enferm UFPE online. 2016;10(8):3149-55. DOI: 10.5205/reuol. 9373-82134-1-RV1008201645

Recebido: 1 de junho de 2020 Aprovado: 16 de junho de 2020

Publicado: 14 de julho de 2020

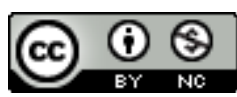

A Revista Baiana de Enfermagem utiliza a Licença Creative Commons - Atribuição-NãoComercial 4.0 Internacional. https://creativecommons.org/licenses/by-nc/4.0/ Este artigo é de acesso aberto distribuído sob os termos da Licença Creative Commons (CC BY-NC). Esta licença permite que outros remixem, adaptem e criem a partir do seu trabalho para fins não comerciais. Embora os novos trabalhos tenham de lhe atribuir o devido crédito e não possam ser usados para fins comerciais, os usuários não têm de licenciar esses trabalhos derivados sob os mesmos termos. 